\title{
A putative helical cytokine functioning in innate immune signalling in Drosophila melanogaster
}

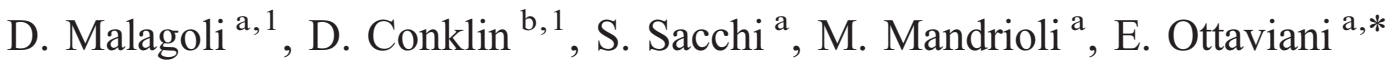 \\ ${ }^{a}$ Department of Animal Biology, University of Modena and Reggio Emilia, via Campi 213/D, 41100 Modena, Italy \\ b Department of Computing, City University, London, UK
}

Received 25 October 2006; received in revised form 21 February 2007; accepted 21 February 2007

Available online 1 March 2007

\begin{abstract}
In invertebrates and vertebrates, innate immunity is considered the first line of defense mechanism against non-self material. In vertebrates, cytokines play a critical role in innate immune signalling. To date, however, the existence of genes encoding for invertebrate helical cytokines has been anticipated, but never demonstrated. Here, we report the first structural and functional evidence of a gene encoding for a putative helical cytokine in Drosophila melanogaster. Functional experiments demonstrate that its expression, as well as that of the antimicrobial factors defensin and cecropin A1, is significantly increased after immune stimulation. These observations suggest the involvement of helical cytokines in the innate immune response of invertebrates.
\end{abstract}

(C) 2007 Elsevier B.V. All rights reserved.

Keywords: Helical cytokine; Insect; Immunity; Antimicrobial peptides

\section{Introduction}

Numerous reports documenting the presence of cytokinelike molecules in invertebrates have been recently reviewed [1]. Immunocytochemical approaches have demonstrated in different invertebrate species belonging to Mollusca, Insecta, Nematoda, Annelida and Tunicata the presence of cytokine-like molecules such as interleukin (IL)-1, IL-2, IL-6, IL-8, tumor necrosis factor- $\alpha$, platelet-derived growth factor (PDGF)-AB, and transforming growth factor (TGF)- $\beta$.

Mammalian cytokines affect invertebrate immune functions, e.g. cell motility, chemotaxis, phagocytosis and cytotoxicity [1]. The cytokines PDGF-AB, TGF- $\beta 1$ and IL-8 induce cell shape changes in molluscan circulating immunocytes throughout the phosphatidylinositol and cAMP pathways [2,3]. PDGF-AB and TGF- $\beta 1$ also provoke a partial inhibition of cell death in the IPLB-LdFB insect cell line, activate the phosphatidylinositol 3kinase, protein kinase $\mathrm{A}$ and protein kinase $\mathrm{C}$ pathways, pro-

\footnotetext{
* Corresponding author. Tel.: +39 059205 5536; fax: +39 0592055548 .

E-mail address: ottaviani.enzo@unimore.it (E. Ottaviani).

${ }^{1}$ These authors contributed equally to this work.
}

mote the wound healing process, and intervene in stress response $[2,4]$. Furthermore, research on IL-2 and corticotrophinreleasing hormone has suggested the presence on molluscan immunocyte membrane of a receptor able to bind both cytokines and neuropeptides [5]. Altogether morphological and functional studies show a close overlap between the humoral factor effects in invertebrates and vertebrates.

Notwithstanding these findings, opposing views persist regarding the presence of cytokines in invertebrates. Mainly on the basis of the limited molecular biological data, it has been hypothesized that invertebrates lack genes orthologous to mammalian cytokines [6]. Though several proteins functioning as cytokines have been found in invertebrates [7-11], conventional bioinformatics approaches cannot detect invertebrate genes orthologous to mammalian helical cytokines, possibly due to evolutionary divergence.

In vertebrates, helical cytokines are a family of structurally related genes, including well-characterized proteins such as IL2 , IL-6, interferon $\alpha-1$ and granulocyte-macrophage colonystimulating factor, and they are distinguished by their unique four helical bundle topology [12]. In this investigation, we utilized a specialized bioinformatics method specifically devel- 
oped to recognize the helical cytokine fold from sequence [12]. This led to the identification and isolation in the Drosophila melanogaster transcriptome of a sequence encoding for a protein that displays structural features indicative of a helical cytokine. This protein will be referred to here as DHF (Drosophila Helical Factor). In addition to the bioinformatics evidence, we have determined that the expression of DHF following immune stimulation implicates the molecule in insect innate immune response.

\section{Materials and methods}

\subsection{Isolation and analysis of helical cytokines in D. melanogaster}

The peptide section of the D. melanogaster genome BDGP4 from the Berkeley Drosophila Genome Project was downloaded from the Ensembl site (www.ensembl.org) October 14, 2005. This section contained 7570 known D. melanogaster protein sequences derived from verified cDNA and EST evidence. An implementation of the QT helical cytokine fold recognition method described by Conklin [12] was applied to these data, looking for sequences which might be indicative of a helical cytokine fold. Secretory signal peptides were predicted by the QT method [12] which employs the von Heijne profiles [13], and signal peptides were also verified by the SignalP method [14] (Version 3.0 at www.cbs.dtu.dk). Considering that many helical cytokines are glycosylated proteins, potential $\mathrm{N}$-linked glycosylation sites were predicted by the NetNGlyc method (Version 1.0 at www.cbs.dtu.dk). The presence of transmembrane domains was explored with the TMHMM method (Version 2.0 at www.cbs.dtu.dk). Coiled-coil motif prediction was performed with the Paircoil server (paircoil.lcs.mit.edu). The QT helical cytokine fold recognition method and various other secondary structure prediction methods in the BioInfoBank Structure Prediction Meta Server at www.bioinfo.pl (namely psipred, sam-t02-dssp, sam-t02-stride and profsec) were applied to the predicted mature peptide, after removal of the predicted signal peptide.

\subsection{Immune stimulation of D. melanogaster larvae}

The flies (FBst0006971, y[1] w[*]; P \{y[Scer|SceI.RS.cRa]=y-donor $\}$ 1B Sb [1]/TM6) used in this investigation were grown on "Formula4-24 ${ }^{\circledR}$ Blue Drosophila" medium (Carolina Biological Supply Company, Burlington, NC 27215, USA) in incubator set at $23 \pm 1{ }^{\circ} \mathrm{C}$. It has been observed that both microinjection and pricking induce a small increase of antimicrobial peptides [15]. Therefore, in consideration of the role that mammalian cytokines play also in wound repair and inflammation, we chose an approach that avoided any possible damage to larval integrity. In lipopolysaccharide (LPS) exposure experiments, 15 third instars larvae were placed in an incubator for $24 \mathrm{~h}$ into $35 \mathrm{~mm}$ tissue culture dish with $1,5 \pm 0.1 \mathrm{~g}$ of medium containing $100 \mu \mathrm{l}$ of $10 \mathrm{U} / \mathrm{ml}(1 \mu \mathrm{g} / \mathrm{ml})$ gel filtration purified Escherichia coli LPS (Sigma, St. Louis, MO, USA). Together with LPS exposure, in order to mimic a natural infection, we adopted also the procedure described by de Morais Guedes et al. [16]. In this case, Drosophila larvae were fed on medium containing a 1:20 proportion of microorganisms' membrane suspension (MMS) (Lantigen B, J07ATX, Bruschettini, Italy). One $\mathrm{ml}$ of MMS contained bacterial lysates from Streptococcus pneumoniae type 3 (63.2 U.A.), Streptococcus pyogenes (group A) (126.2 U.A.), Branhamella catarrhalis (39.9 U.A.), Staphylococcus aureus (79.6 U.A.), Haemophylus influentiae (type b) (50.2 U.A.) and Klebsiella pneumoniae (39.8 U.A.). For both LPS and MMS exposures only distilled water was added to the food of control larvae. Each experiment was repeated in duplicate for three times.

\subsection{Immune stimulation of SL2 cell line}

Drosophila embryonic hemocytes (SL2 cell line) were maintained at $25^{\circ} \mathrm{C}$ in Schneider medium (Sigma), supplemented with heat-inactivated 10\% FCS. In LPS and MMS exposure experiments, $10^{6}$ cells were suspended in $5 \mathrm{ml}$ of medium containing either $10 \mathrm{U} / \mathrm{ml}$ gel filtration purified $E$. coli LPS (Sigma) or $250 \mu 1$ Lantigen B (Bruschettini). SL2 cells were subjected to a 24-h treatment, before RNA extraction. Each experiment was repeated in duplicate for three times.

\subsection{Sequence amplification, sequencing and analysis of dhf}

A coding sequence for D. melanogaster DHF was used to design the following pair of primers for use in RT-PCR reactions for amplification with either larval or hemocyte cDNA as a template: DHF_F 5'-AGT GAA AGA ACG CAG CCC TA-3' and DHF_R 5'-AAC TGG GTG ACG TTG GAA AC-3'. Cytoplasmic actin expression was evaluated as loading control (see below). After LPS or MMS exposure, the larvae were collected, washed in sterile phosphatebuffered saline (PBS) $\left(137 \mathrm{mM} \mathrm{NaCl}, 2.7 \mathrm{mM} \mathrm{KCl}, 10 \mathrm{mM} \mathrm{Na}_{2} \mathrm{HPO}_{4}, 10 \mathrm{mM}\right.$ $\mathrm{NaH}_{2} \mathrm{PO}_{4}$, pH $7.3 \pm 0.1$ ), centrifuged for $3 \mathrm{~min}$ at $100 \times g$ and total RNA was extracted using TRI REAGENTTM (Sigma), following the method described by the supplier. SL2 cells were centrifuged for $10 \mathrm{~min}$ at $800 \times \mathrm{g}$ in order to remove the culture medium then total RNA was extracted as indicated above for larvae. cDNA of both larvae and SL2 cells was obtained using "RevertAid H Minus First Strand cDNA Synthesis Kit" (Fermentas AB, Vilnius, Lithuania) using $2 \mu \mathrm{g}$ of total RNA as template. Semiquantitative PCR reactions for $d h f$ were performed with an annealing temperature of $54^{\circ} \mathrm{C}$; time of annealing was $60 \mathrm{~s}$ and the time of elongation was set at $60 \mathrm{~s}$. The number of PCR cycles was set in order to maintain the reaction within the exponential phase, in this case between 25 and 35 cycles. Amplified fragment was extracted from agarose gel and purified with "QIAquick ${ }^{\circledR}$ Gel Extraction Kit" (QIAGEN GmBH, Hilden, Germany). The correspondence between the expected and the amplified fragment was confirmed through sequencing performed by "Servizio Sequenziamento Automatico-Progetto Camilla" (Pomezia, Italy). Sequencing reactions were performed with primers DHF_F and DHF_R. The obtained sequences were confirmed by sequence alignment to correspond to the expected $d h f$ coding sequence. In order to exclude any false positive results due to the presence of contaminating genomic DNA, negative control reactions were realized either by substituting for cDNA an equal amount of purified RNA or by replacing template with molecular biology grade water (Sigma).

\subsection{Sequence amplification, sequencing and analysis of defensin, cecropin Al and cytoplasmic actin}

In order to demonstrate that the applied experimental conditions were able to elicit an immune response in D. melanogaster larvae and embryonic hemocytes, the expression of defensin [17] (F 5'-GCT ATC GCT TTT GCT CTG CT- $3^{\prime}$ and R 5'-CCA CTT GGA GAG TAG GTC GC-3') and cecropin A1 [18] (F 5'-ACA TCT TCG TTT TCG TCG CT- ${ }^{\prime}$ and R $5^{\prime}$-CTT GTT GAG CGA TTC CCA GC- $3^{\prime}$ ) was evaluated. Cytoplasmic actin (F $5^{\prime}$-AGC AGG AGA TGG CCA CC-3' and R 5'-TCC ACA TCT GCT GGA AGG-3') expression was evaluated as loading control. For defensin and cecropin A1 semiquantitative PCR reactions (between 30 and 45 cycles) the following parameters were used: annealing temperature $62{ }^{\circ} \mathrm{C}$; time of annealing $60 \mathrm{~s}$, time of elongation $30 \mathrm{~s}$; for cytoplasmic actin semiquantitative PCR reactions (between 15 and 25 cycles) the following parameters were used: annealing temperature $58{ }^{\circ} \mathrm{C}$; time of annealing $40 \mathrm{~s}$, time of elongation $45 \mathrm{~s}$. The amplified fragments of defensin, cecropin A1 and cytoplasmic actin were extracted from agarose gel, sequenced and compared to expected sequences as indicated for $d h f$. Negative control reactions were performed as described above for $d h f$.

\section{Results and discussion}

An implementation of a fold recognition method for helical cytokines [12], referred to in this study as the QT method, was performed on a database of 7570 known D. melanogaster protein sequences. The QT method indicated that the highestscoring peptide in the helical cytokine search is the sequence referenced as GenPept accession no. AAF53861. This sequence was originally deposited by Jagadeeshan and Singh [19], and is annotated with the definition "olfactory-specific protein Os9". However, subsequent searches of Entrez with 
the term OS9 revealed additionally an entirely different D. melanogaster gene named OS9, cloned by Raha and Carlson [20] and deposited as GenPept accession no. AAB31188. The ambiguity of the OS9 designation led us to refer to the GenPept accession no. AAF5 3861 with the new name DHF (Drosophila Helical Factor). DHF is a peptide of 214 amino acids, and the QT method predicts that this sequence has a helical cytokine fold with four core amphipathic helices placed as indicated in Fig. 1. The overall secondary structure has in addition been verified by several other prediction methods to be predominately helical (Fig. 1). The sequence is not predicted to contain transmembrane domains and this is consistent with the QT prediction of a secreted globular helical protein. The putative signal peptide (Fig. 1) comprising Met1 through Ser32 was predicted by the QT method, which employs the von Heijne profiles [13], and was also verified by the SignalP method. The DHF protein without the signal peptide has a predicted $\mathrm{pI} /$ Mw of 6.72/21000. The $d h f$ gene resides on $D$. melanogaster chromosome arm $2 \mathrm{~L}$ and its coding sequence resides on 2 exons separated by a short intron of $63 \mathrm{bp}$. The exon/intron junction is within the predicted signal peptide region and is in phase 0 . In terms of homology, BLAST searches reveal no similar sequences other than DHF orthologues from other species of the genus Drosophila; particularly, D. simulans
(95\% identity), D. yakuba (87\%) and D. pseudoobscura (61\%). The DHF protein is predicted by the NetNGlyc method to have 2 N-linked glycosylation sites at Asn76 and Asn160 (Fig. 1). The glycosylation motif $\mathrm{NX}[\mathrm{S} / \mathrm{T}]$ is conserved at corresponding positions within D. simulans and D. yakuba; in D. pseudoobscura the Asn76 site is not conserved, though an alternative nearby glycosylation site is predicted. The DHF peptide is not predicted by Paircoil to contain extended coiled-coil amphipathic helical motifs incompatible with the loop topology of the helical cytokines.

All fold recognition approaches, when calibrated for a zero false negative rate, will produce false positive results: for the QT method these will be proteins that are predominately helical in structure though without a helical cytokine topology. Within the 7570 known D. melanogaster proteins, we find two secreted proteins with high QT scores (though below the score of DHF): the products of genes Acp26Aa (accessory gland-specific peptide 26Aa), and Obp19a (odorant-binding protein 19a). Containing several pairs of basic amino acids, Acp26Aa is a precursor to multiple peptide hormones [21] and is unlikely to also function without posttranslational processing as a helical cytokine. A helical fold for Obp19a can be inferred by homology to Obp76a (Protein Data Bank 1OOH) though it does not have the helical cytokine topology.

a)

$\begin{array}{lccc}1 & 10 & 20 & 30 \\ \text { MARSGRSSRFLIGGSTCCSMLLGLICLALADS }\end{array}$

b)

$\begin{array}{llllll}40 & 50 & 60 & 70 & 80 & 90\end{array}$

SQVKERSPTILVRNTIQAVERLFNQVLYTTIEDARQRLPGLPANETIDRQYFEELDLVAG

1. - - - - - - -

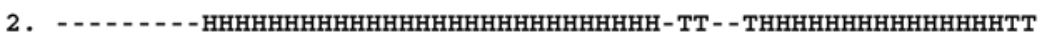

3. - - TT - - - HHHHHHHHHHHHHHHHHHHHHHHHHHHHTTTT - TTHHHHHHHHHHHHHHT -

4. - - - - EEEEE - HHHHHHHHHHHHHH - HHHHHHH - - - - - - -1

5.

$\begin{array}{llllll}100 & 110 & 120 & 130 & 140 & 150\end{array}$

NEDYYSTAFYIFAWINSDLMYHKTPDKLLVEVLPGEKIAIRRFFAKVKPHLTKYLRLSRQ

1. - - - HHHHHHHHHHHHH - - $\mathrm{HHH}-\mathbf{-}-\mathbf{-}$ HHHHHHH - - - - HHHHHHHHHHHHHHHHHHHHHHHH

2. - - - HHHHHHHHHHHHHHHHHHH - - TTEEEEEE - TTHHHHHHHHHHHH - HHHHHHHHHHHH

3. TTTHHHHHHHHHHHHHHHHHHTTTTTTEEEEETTTHHHHHHHHHHHH - HHHHHHHHHHHH

4. - - - EEEEEHHHHHHHH - - - - - - - - - - - -

5. - - - HHHHHHHHHHHHHHH - -

$\begin{array}{llllll}160 & 170 & 180 & 190 & 200 & 210\end{array}$

DRSELVSNVTTLATETKDSLITTFLEFPQNLKSQLPELQLMDYTKLAQALVQGVASGIWTKA

1. HHHHHHHHHHHHHHHHHHHHHHHHHHHHHHHHH - - - - HHHHHHHHHHHHHHHHHHHH - -

2. HHHHHHHHHHHHHTH - - - EEEEEEEE - - HHHHHHS - HGHHHHHHHHHHHHHHHHHTTEEE

3. - HHHHHHHHHHHHHHTTTEEEEEEEE - HHHHHHHHHHHHHHHHHHHHHHHHHHHHH - E- -

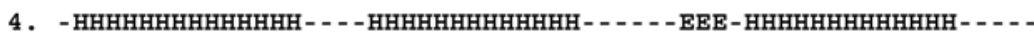

5.

Fig. 1. (a) Signal peptide of DHF predicted with the QT method. The predicted signal peptide is Met1 through Ser32. (b) Structure predictions of DHF performed with the QT helical cytokine fold recognition method and various other secondary structure prediction methods. The following secondary structure prediction approaches were applied to the predicted mature peptide (Ser33 through Ala214): 1 psipred; 2 sam-t02-dssp; 3 sam-t02-stride; 4 profsec; 5. QT (H: helix, T: turn, E: strand). The two predicted NX[S/T] glycosylation motifs at Asn76 and Asn160 are indicated in boxes. 
As far as functional evidence is concerned, RT-PCR experiments performed on larvae exposed to $10 \mathrm{U} / \mathrm{ml}$ of LPS revealed a significant increase in $d h f$ expression and antimicrobial peptides (Figs. 2, 3), but no increase in expression was observed after feeding with MMS (data not shown). Conversely, MMS stimulated dhf and antimicrobial peptide expression in SL2 cells (Fig. 4), while no induction was seen after treating the cells with LPS (data not shown). Experiments performed by other groups have demonstrated that microinjections of LPS in $D$. melanogaster larvae promote the synthesis of both defensin [22] and cecropin [23]. Regarding defensin synthesis, Lemaitre et al. [15] observed that the Gram-negative and Drosophila pathogen Serratia marcescens does not induce antimicrobial peptides in adult flies when introduced into the food. However, the insects die within days as a consequence of the treatment. In larvae of D. melanogaster, it has been established that the synthesis of several peptides is increased after feeding with MMS, but neither defensins nor cecropins are reported to be induced by the treatment [16]. Accordingly, we observe here that feeding larvae with MMS does not affect defensin and cecropin expression, while LPS-containing medium provokes an immune stimulation as indicated by the induction of defensin and cecropin A1 (Fig. 3). In this context, it is possible that the induction we have observed as a consequence to LPS exposure is due to peptidoglycan impurities contained in our commercial preparation [24]. This should be taken into account for studies devoted to the unravelling of the pathway involved in $d h f$ expression.

The concomitant induction of $d h f$ and antimicrobial peptides after LPS feeding suggests a relationship between the predicted fly helical cytokine and innate immune response. This conclusion relies also on the observation that SL2 cells increase $d h f$ expression together with that of defensin and cecropin A1 after exposure to MMS. Furthermore, the mRNA for the DHF peptide has also been isolated by an EST sequence from fat body of third instar larvae challenged with Gram positive and negative bacteria (see GenBank accession no. CO193348). The expression in an immune-related organ, and after Gram-positive and

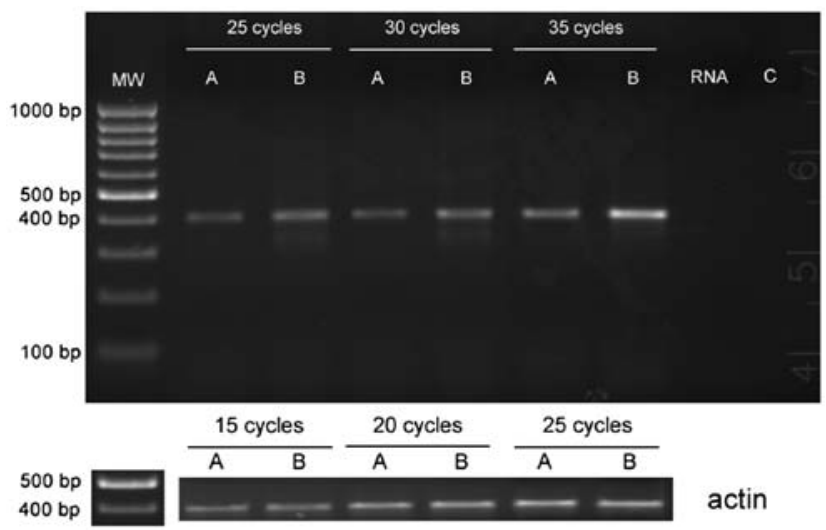

Fig. 2. Evaluation by RT-PCR of $d h f$ expression after $24 \mathrm{~h}$ LPS exposure in D. melanogaster third instar larvae. The number of cycles for semiquantitative RT-PCR reactions are reported. Expression of cytoplasmic actin has been used as loading control. $\mathrm{A}=$ control; $\mathrm{B}=10 \mathrm{U} / \mathrm{ml} \mathrm{LPS}$; RNA=amplification with RNA instead of cDNA ( 35 cycles); $\mathrm{C}=$ negative control with water instead of template (35 cycles).

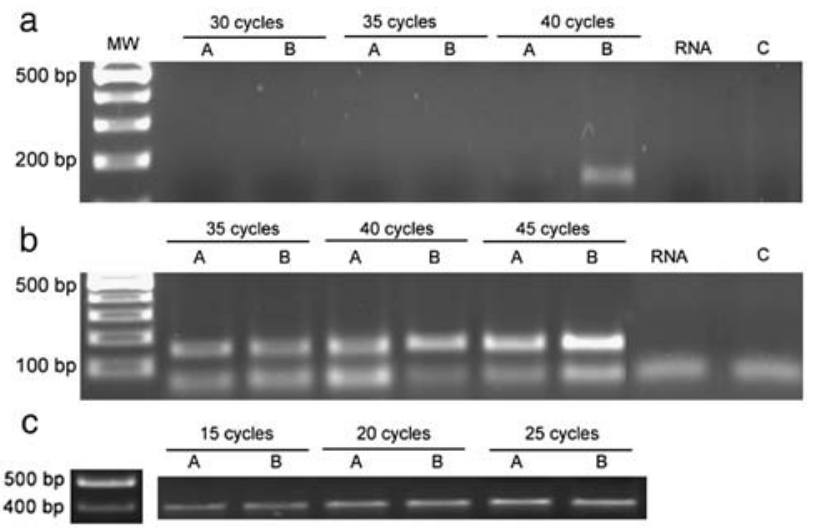

Fig. 3. Evaluation by RT-PCR of defensin (a) and cecropin Al (b) expression after $24 \mathrm{~h}$ LPS exposure in D. melanogaster third instar larvae. The number of cycles for semiquantitative RT-PCR reactions are reported. Expression of cytoplasmic actin (c) has been used as loading control. $\mathrm{A}=$ control; $\mathrm{B}=10 \mathrm{U} / \mathrm{ml} \mathrm{LPS}$; RNA=amplification with RNA instead of cDNA (35 cycles); $\mathrm{C}=$ negative control with water instead of template ( 35 cycles).

Gram-negative challenge, strengthen our hypothesis of an involvement of $d h f$ in immunity.

Genes orthologous to mammalian helical cytokines have been identified in teleost fish but so far have not been reported in invertebrates [25]. Though the D. melanogaster genome encodes a protein belonging to the class I cytokine receptor family, with this receptor activated by the Unpaired (UPD) protein [7], from the results obtained with the QT method there is no evidence to indicate that UPD has a helical cytokine structure.

In the present investigation, we utilized the specialized QT fold recognition method for the identification of a conserved structure rather than a conserved sequence. The bioinformatics results presented here indicate that DHF has features consistent with that of helical cytokines. Our functional experiments indicate that $d h f$ gene expression is significantly increased as a consequence of LPS ingestion by third instar larvae and after MMS exposure by SL2 embryonic hemocytes. In both cases, the increase in $d h f$ expression is concomitant with the induction of defensin and cecropin A1, and this is consistent with a possible role for this molecule in immune functions. Obviously,

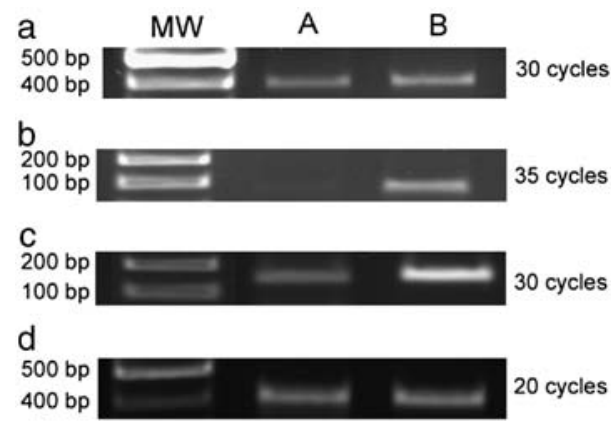

Fig. 4. Evaluation by RT-PCR of $d h f$ (a) defensin (b) and cecropin Al (c) expression after $24 \mathrm{~h}$ MMS exposure of SL2 embryonic hemocytes of D. melanogaster. The bands are representative of semiquantitative RT-PCR reactions and the number of cycles corresponding to the maximum difference between control and treated samples is reported. Expression of cytoplasmic actin (d) has been used as loading control. $\mathrm{A}=$ control; $\mathrm{B}=1: 20 \mathrm{MMS}$. 
a crystal structure and a full characterization of the functions played by DHF is needed at this stage before definitely concluding that it has roles comparable to those of a mammalian cytokine.

\section{Acknowledgements}

The authors are grateful to Prof. S. Calandra (University of Modena Reggio Emilia, Italy) who kindly gifted Drosophila embryo SL2 cells. This work was supported by a MIUR (Italy) grant to E.O.

\section{References}

[1] E. Ottaviani, D. Malagoli, A. Franchini, Invertebrate humoral factors: cytokines as mediators of cell survival, Prog. Mol. Subcell. Biol. 34 (2004) $1-25$.

[2] E. Ottaviani, A. Franchini, D. Kletsas, PDGF and TGF- $\beta$ in invertebrate immune and neuroendocrine interactions: another sign of conservation in evolution, Comp. Biochem. Physiol. 129C (2001) 295-306.

[3] E. Ottaviani, A. Franchini, D. Malagoli, S. Genedani, Immunomodulation by recombinant human interleukin-8 and its signal transduction pathways in invertebrate hemocytes, Cell. Mol. Life Sci. 57 (2000) 506-513.

[4] E. Ottaviani, D. Barbieri, D. Malagoli, D. Kletsas, Involvement of PI 3-kinase, PKA and PKC in PDGF- and TGF- $\beta$-mediated prevention of 2-deoxy-D-ribose-induced apoptosis in the insect cell line, IPLBLdFB, Cell Biol. Int. 25 (2001) 171-177.

[5] E. Ottaviani, A. Franchini, E. Caselgrandi, A. Cossarizza, C. Franceschi, Relationship between corticotropin-releasing factor and interleukin-2: evolutionary evidence, FEBS Lett. 351 (1994) 19-21.

[6] A. Beschin, M. Bilej, L. Brys, E. Torreele, R. Lucas, S. Magez, P. De Baetselier, Convergent evolution of cytokines, Nature 400 (1999) 627-628.

[7] D.A. Harrison, P.E. McCoon, R. Binari, M. Gilman, N. Perrimon, Drosophila unpaired encodes a secreted protein that activates the JAK signaling pathway, Genes Dev. 12 (1998) 3252-3263.

[8] P.M. Hermann, R.E. van Kesteren, W.C. Wildering, S.D. Painter, J.M. Reno, J.S. Smith, S.B. Kumar, W.P. Geraerts, L.H. Ericsson, A.B. Smit, A.G. Bulloch, G.T. Nagle, Neurotrophic actions of a novel molluscan epidermal growth factor, J. Neurosci. 20 (2000) 6355-6364.

[9] D.B. Akalal, G.T. Nagle, Mollusk-derived growth factor: cloning and developmental expression in the central nervous system and reproductive tract of Aplysia, Brain Res. Mol. Brain Res. 91 (2001) 163-168.
[10] H. Agaisse, N. Perrimon, The roles of JAK/STAT signaling in Drosophila immune responses, Immunol. Rev. (2004) 72-82.

[11] A. Herpin, C. Lelong, P. Favrel, Transforming growth factor- $\beta$-related proteins: an ancestral and widespread superfamily of cytokines in metazoans, Dev. Comp. Immunol. 28 (2004) 461-485.

[12] D. Conklin, Recognition of the helical cytokine fold, J. Comput. Biol. 11 (2004) 1189-1200

[13] G. von Heijne, A new method for predicting signal sequence cleavage sites, Nucleic Acids Res. 14 (1986) 4683-4690.

[14] J. Bendtsen, H. Nielsen, G. von Heijne, S. Brunak, Improved prediction of signal peptides: SignalP 3.0, J. Mol. Biol. 340 (2004) 783-795.

[15] B. Lemaitre, J.-M. Reichart, J.A. Hoffmann, Drosophila host defense: differential induction of antimicrobial peptide genes after infection by various classes of microorganisms, Proc. Natl. Acad. Sci. U. S. A. 94 (1997) 14614-14619.

[16] S. de Morais Guedes, R. Vitorino, R. Domingues, K. Tomer, A.J.F. Correia, F. Amado, P. Domingues, Proteomics of immune-challenged Drosophila melanogaster larvae hemolymph, Biochem. Biophys. Res. Commun. 328 (2005) 106-115.

[17] B.P. Lazzaro, A.G. Clark, Molecular population genetics of inducible antibacterial peptide genes in Drosophila melanogaster, Mol. Biol. Evol. 20 (2003) 914-923.

[18] A.G. Clark, L. Wang, Molecular population genetics of Drosophila immune system genes, Genetics 147 (1997) 713-724.

[19] S. Jagadeeshan, R.S. Singh, Rapidly evolving genes of Drosophila: differing levels of selective pressure in testis, ovary, and head tissues between sibling species, Mol. Biol. Evol. 22 (2005) 1793-1801.

[20] D. Raha, J. Carlson, OS9: a novel olfactory gene of Drosophila expressed in two olfactory organs, J. Neurobiol. 25 (1994) 169-184.

[21] M.F. Wolfner, The gifts that keep on giving: physiological functions and evolutionary dynamics of male seminal proteins in Drosophila, Heredity 88 (2002) 85-93.

[22] Y.-S. Kim, S.-J. Han, J.-H. Ryu, K.-H. Choi, Y.-S. Hong, Y.-H. Chung, S. Perrot, A. Raibaud, P.T. Brey, W.-J. Lee, Lipopolysaccharide-activated kinase, an essential component for the induction of the antimicrobial peptide genes in Drosophila melanogaster cells, J. Biol. Chem. 3 (2000) 2071-2079.

[23] E. Roos, G. Björklund, Y. Engström, In vivo regulation of tissue-specific and LPS-inducible expression of the Drosophila Cecropin genes, Insect Mol. Biol. 7 (1998) 51-62.

[24] T. Kaneko, W.E. Goldman, P. Mellroth, H. Steiner, K. Fukase, S. Kusumoto, W. Harley, A. Fox, D. Golenbock, N. Silverman, Monomeric and polymeric gram-negative peptidoglycan but not purified LPS stimulate the Drosophila IMD pathway, Immunity 20 (2004) 637-649.

[25] M. Huising, C. Kruiswijk, G. Flik, Phylogeny and evolution of class-I helical cytokines, J. Endocrinol. 189 (2006) 1-25. 